\title{
Factors relating to home telehealth acceptance and usage compliance
}

\author{
This article was published in the following Dove Press journal: \\ Risk Management and Healthcare Policy \\ 19 April 2012 \\ Number of times this article has been viewed
}

\author{
Rachael Wade \\ Colleen Cartwright \\ Kelly Shaw \\ ASLaRC Aged Services Unit, Health \\ and Wellbeing Research Cluster, \\ Southern Cross University, Coffs \\ Harbour, NSW, Australia
}

Aim: This paper investigates the acceptance of in-home telehealth by frail older adults and carers of the Transition Care Program (TCP), and evaluates telehealth acceptance as a predictor for usage compliance.

Method: A stratified random sample of participants was allocated to one of five groups: either a control group or to receive telehealth monitoring of their vital signs for a period of 12 or 24 weeks; with or without a medical alarm pendant.

Results: Before being trained in and using telehealth, the majority of participants and carers demonstrated acceptance of the technology by reporting that they perceived it would be "useful" and "easy to use." This acceptance was also reported post-TCP (up to 12 weeks of usage). The "perceived ease of use" of the telehealth equipment increased significantly from pre-telehealth training and usage to post-TCP (up to 12 weeks of usage) $(P=0.001)$. There was no change, (pre-training and usage to post-TCP) in the "perceived usefulness" of the telehealth equipment. The telehealth acceptance constructs of "ease of use" and "usefulness," at pre-telehealth training and usage, approached statistical significance as a predictor of future compliance $(P=0.06)$. "Perceived ease of use," at pre-training and usage, had a positive relationship with future compliance $(P=0.02)$.

Conclusion: There is currently limited knowledge about the influences and determinants of home telehealth compliance in frail older people and their carers, potentially a significant user group for the technology into the future. This study's finding that frail older people and their carers perceive that home telehealth is useful and easy to use demonstrates their acceptance of home telehealth as a therapeutic tool. Further, perceived ease of use of home telehealth is a significant predictor of compliance with frail older people and their carers' use of home telehealth. Additional research is required in order to identify other influences and determinants of home telehealth compliance with this group. Knowledge about the influences and determinants of home telehealth compliance may assist the development of targeted interventions aimed at encouraging high compliance with users who are recording lower reading rates.

Keywords: safety, older people, technology acceptance model, quasi-randomized controlled trial

\section{Introduction}

Heath care systems in Australia will be challenged in the future by an increase in the numbers and expectations of older people, a relative decline in informal carers and the need for a larger health care workforce. ${ }^{1}$ The Productivity Commission has proposed that these weaknesses be addressed by promoting independence, connectedness, and choice for older Australians. Under the proposed reforms, older Australians would receive a flexible range of care and support services that meet their individual needs, 
including where feasible and appropriate, a choice to receive care at home. ${ }^{1}$ Thus, there is a policy imperative to improve older people's ability to remain within their own homes as long as possible, with access to care and support services that enable them to do this safely.

Telehealth, the remote monitoring of clients' health ${ }^{2}$ is currently being trialed as one possible option for this to occur. Numerous studies have reported an improvement in patient satisfaction and the quality of patient care with home telehealth; ${ }^{3-5}$ however, few have investigated safety issues. A systematic review of safety in home telehealth concluded that there was a paucity of home telehealth research relating to safety and recommended that further research be undertaken in this area. ${ }^{6}$ A recent Australian study exploring home telehealth usage and acceptance reported several contributing factors to the safety aspect of home telehealth reading failure rate. These included staff not following up, equipment failure, participants not returning a call from staff investigating nonreading, user error, and participant noncompliance. ${ }^{\text {? }}$

The importance of medical compliance is well documented. Patient compliance with medical regimes is reported as a factor in improving health outcomes. ${ }^{8}$ Conversely, noncompliance can reduce the quality of health care, increase health care costs, and in some circumstances, lead to negative health outcomes. ${ }^{9}$ Interventions to increase medical compliance generally focus on educating patients; however, unless the determinants of poor compliance are known, an intervention is unlikely to be successful in increasing compliance rates. ${ }^{9}$

The determinants of telehealth compliance are poorly understood..$^{10}$ User acceptance of telehealth has been suggested as a possible determinant of telehealth compliance. ${ }^{11}$ The technology acceptance model (TAM) is a theoretical framework that explores user acceptance and usage of technology, and recently, user acceptance and usage of telehealth. ${ }^{12}$ TAM proposes that the constructs of perceived equipment ease of use and usefulness are primary factors in technology user acceptance and usage. Ease of use is defined as the degree to which using the equipment is free from effort, and usefulness as the extent to which the person believes that the technology will assist them. ${ }^{13}$ In essence, TAM states that the more positive the perceptions of ease of use and usefulness are, the more accepting a person is of that form of technology, and the more likely that they will comply with using it. ${ }^{14}$

A small body of telehealth research has utilized TAM to demonstrate a relationship between acceptance of, and intention to use, telehealth. ${ }^{12,15,16}$ Few studies have investigated the relationship between user acceptance and usage compliance of home telehealth. ${ }^{11}$

\section{Aim}

The findings presented in this paper are part of a larger study investigating acceptance and usage of telehealth products by frail older clients of the Transition Care Program (TCP) and their carers. This paper reports participant and carer acceptance of in-home telehealth, and evaluates telehealth acceptance as a predictor for usage compliance.

\section{Methods}

This study was approved by Southern Cross University Human Research Ethics Committee and Baptist Community Services (BCS) Human Research Ethics Committee.

\section{Target group}

The target group was frail older clients from BCS communitybased Transition Care Program who were being discharged from hospital to home, had a chronic disease, and were at risk of being admitted into residential care. The BCS sites were in urban and regional Eastern Australia. Carers of participating clients were also invited to take part in the study.

\section{Recruitment period}

The project recruited participants over a period of 14 months to October 2010.

\section{Design}

The study design was a quasi-randomized controlled clinical trial. A stratified random sample of participants was allocated to one of five groups using a random number table. BCS recruitment staff at each site were provided with packages numbered 1-5, containing the relevant information sheet and baseline questionnaire for each group, and a random number list, with the numbers 1-5 in continuous random order. Study participants who were already in possession of a pendant alarm provided by the study service provider at study commencement, or were assessed as needing a pendant alarm, were allocated to the first number out of 1 (control group) or 4 or 5 (home telehealth monitoring with a pendant alarm up to 12 or 24 weeks respectively) on the random number table list for that site, and the number was crossed off the list. If they were in possession of a pendant alarm that was not from the study service provider they were automatically allocated to the control group and the next number 1 on the random number table was crossed off. If they were assessed as not needing a pendant alarm, they were allocated to the first 
number on the list out of 2 or 3 (home telehealth monitoring up to 12 or 24 weeks respectively); again the number was crossed off the list.

The number of participants and carers allocated to each group at baseline; a summary of the duration of the telehealth monitoring for each group condition; provision of a pendant alarm; and the telehealth monitoring peripherals requested by participant's general practitioner (GP) are outline in Table 1.

\section{Intervention}

Group 1 comprised non-telehealth controls and received usual transition care. Groups 2 and 3 received transition care plus telehealth monitoring of blood pressure, heart rate, oxygen saturation levels, and bodyweight for 12 weeks or 24 weeks respectively. Groups 4 and 5 received telehealth monitoring (as described above) and also used a pendant alarm for 12 weeks or 24 weeks respectively.

Three separate sets of questionnaires (baseline, midpoint, and final) were also completed by participants. (Note: there were separate questionnaires for clients and carers). This paper will report the data for the attitudes and acceptance section of the telehealth questionnaires up to the end of the Transition Care Program (midpoint - up to 12 weeks of telehealth monitoring).

\section{Client exclusion criteria}

The following exclusion criteria were applied:

1. The client lacked capacity to complete surveys and/or operate the equipment, and had no-one to do it for them. Individuals with cognitive impairment were only excluded from the study if they did not have a carer. Where the person lacked capacity but had someone to assist them with the equipment and the readings, they were not excluded.

2. The client had a carer, and the carer did not wish the client to participate.
3. The client had a live-in carer, and the client did not want the carer involved.

4. There was no phone line connected to the house, or there was no power source in a suitable location in the house or nowhere to install the equipment, which would not be a hazard to the client or potentially result in damage to the equipment.

5. The client was using other telehealth products before going into hospital and intended to continue using those products.

6. The client's live-in carer or partner was already participating in the project.

7. The client's live-in carer or partner was already using telehealth equipment.

\section{Equipment}

Participants in groups 2-5 were provided with a Tunstall ${ }^{\circledR}$ (Tunstall Healthcare, Yorkshire, UK) home monitoring system. All telehealth monitored participants, or their carers, were required to complete eleven telehealth-monitored questions on a daily basis; four questions related to physical functioning; two to emotional state; one to care and support; and four to service use. Participants responded to questions by pressing either yes or no on the telehealth monitor.

Four peripherals accompanied the telehealth equipment; these measured weight, blood pressure, heart rate, and oxygen saturation. Peripheral parameters were determined by the client's GP prior to commencing the study. A trained staff member visited all participants receiving the telehealth equipment in the client's home, installed the equipment, and trained the client (and carer where applicable) in its use. Participants were also encouraged to phone for additional support if required. Operational proficiency of the equipment was assessed by a staff member of the participating organization who was responsible for providing participants with the equipment and instructing them in its use.

Table I Number of participants and carers allocated to each group at baseline; summary of duration of telehealth monitoring for each group condition, provision of pendant alarm, and telehealth monitoring requested by general practitioner

\begin{tabular}{|c|c|c|c|c|c|c|c|c|}
\hline \multirow[t]{2}{*}{ Group } & \multicolumn{2}{|l|}{$\mathbf{N}$} & \multirow{2}{*}{$\begin{array}{l}\text { Monitoring } \\
\text { duration }^{\mathrm{a}}\end{array}$} & \multirow[t]{2}{*}{ Pendant } & \multirow[t]{2}{*}{ Weight } & \multirow[t]{2}{*}{ Oxygen } & \multirow[t]{2}{*}{$\mathbf{B P}$} & \multirow{2}{*}{$\begin{array}{l}\text { Heart } \\
\text { rate }\end{array}$} \\
\hline & $\mathbf{P}$ & $\bar{c}$ & & & & & & \\
\hline Control/group I & 16 & 5 & $\mathrm{a}$ & 10 & N/A & N/A & N/A & N/A \\
\hline Group 2 & 6 & 4 & $\mathrm{a}$ & N/A & I & 2 & 6 & 6 \\
\hline Group 3 & 9 & 4 & $b$ & N/A & 2 & 2 & 8 & 9 \\
\hline Group 4 & 16 & 7 & $\mathrm{a}$ & 16 & 10 & 8 & 16 & 16 \\
\hline Group 5 & 14 & 9 & $b$ & 14 & 6 & 5 & 14 & 14 \\
\hline
\end{tabular}

Notes: ${ }^{a} \mathrm{a}=$ duration TCP (up to 12 weeks); $\mathrm{b}=$ duration TCP plus additional 12 weeks.

Abbreviations: BP, blood pressure; C, carer; N/A, not applicable; P, participant; TCP, Transition Care Program. 


\section{Telehealth acceptance questionnaire}

Participants who were monitored by telehealth, (and their carer if they had one), each completed a separate telehealth acceptance questionnaire prior to being trained in and using the equipment, at completion of TCP (up to 12 weeks of telehealth usage), and 12 weeks post-TCP completion. The acceptance questionnaire was based on the TAM. The questionnaire had two scales: (1) the telehealth equipment's perceived usefulness scale; and (2) perceived ease-of-use scale. Each scale contained four questions requiring the participant to respond on a five-point Likert scale $(1=$ strongly agree, $2=$ agree, $3=$ neutral, $4=$ disagree, $5=$ strongly disagree).

The perceived usefulness questions (with wording varied to reflect the client or carer version of the questionnaire, eg, "regular testing of my health condition" or "regular testing of the health condition of the person I care for") were:

- "using the telehealth equipment will improve access to regular testing of my health condition;"

- "using the telehealth equipment will make it easier to do regular testing;"

- "using the telehealth equipment will save time in having regular testing;" and

- "I will find the telehealth equipment useful in my regular testing."

The perceived ease of use questions were:

- "learning to operate the telehealth equipment will be easy for me;"

- "my interaction with the telehealth equipment will be clear and understandable;"

- "it will be easy for me to become skillful at using the telehealth equipment;" and

- "I will find the telehealth equipment easy to use."

\section{Data collation and analysis}

At each required stage, the participant, or their carer if they had one, completed the telehealth acceptance questionnaire and returned it to BCS. The BCS staff then forwarded the questionnaires on to the research team, where they were coded and analyzed using SPSS Statistics (IBM Corporation, Somers, NY) software, version 17.

Data from the telehealth equipment were provided by Tunstall to BCS, where it was transcribed into a Microsoft Excel spreadsheet. Readings outside the set parameters were faxed to the client's GP on each occasion. Incomplete or missed readings were followed up by the BCS data monitoring officer who contacted the client/carer and recorded the reason for the missed or incomplete reading. At the completion of the monitoring period, data were analyzed descriptively using SPSS software.

\section{Results}

There were 255 people eligible for post-acute care who were approached to participate in the study. Of those, $112 \mathrm{did}$ not meet the inclusion criteria for participation: 50 were already using another form or brand of telehealth equipment and wanted to continue to do so; 32 lacked sufficient capacity and did not have a carer who could assist them with their readings on a regular basis (BCS staff observationally assessed those participants as not having the physical and/or cognitive capacity to operate the telehealth equipment, complete questionnaires, and to report any issues to staff); nine had insufficient English to participate; and for the remaining 21 people, recruiting staff did not provide a reason for exclusion. Of the 143 people who did meet the inclusion criteria, 76 declined to participate, four were readmitted to hospital, and two withdrew prior to being randomized into groups, leaving 61 participants who were enrolled and randomized into groups. There were 29 carers who were eligible to participate; all gave consent and were enrolled in the study.

Clients were randomized to groups, and carers were allocated into the same group as the client they cared for. Sixteen clients and five carers were randomized into the control group; six clients and four carers were randomized into group 2; nine clients and four carers were randomized into group 3; 16 clients and seven carers were randomized into group 4; and 14 clients and nine carers were randomized into group 5 (ie, 61 clients and 29 carers).

Of those enrolled, 42 clients and 19 carers completed the study ( $68 \%$ completion rate). Of those who completed, eleven clients and four carers were in the control group, four clients and one carer were in group 2, four clients and one carer were in group 3, eleven clients and five carers were in group 4, and twelve clients and eight carers were in group 5.

For those who did not complete the study ( $\mathrm{n}=19$ clients, of whom $n=10$ had a carer), the reasons for noncompletion were as follows: $47 \%(n=9)$ were admitted to hospital; $26 \%(n=5)$ no longer wanted to be part of the study; $16 \%(n=3)$ had a carer who no longer wanted the client to be part of the study; and $11 \%(n=2)$ went into residential care.

All participants without a carer who were monitored by telehealth undertook their own telehealth monitoring and also completed the study questionnaires. For all participants with a carer, the carer undertook the telehealth monitoring; among the "cared for" participants, all carers and all but two 
participants (those two lacked capacity to do so) completed the study questionnaires.

This paper reports the data for those who used the telehealth equipment (participants without a carer and carers of participants in groups 2, 3, 4, or 5), and completed a telehealth acceptance questionnaire prior to receiving the telehealth training and equipment, and also at the completion of their TCP (up to 12 weeks of telehealth).

The telehealth acceptance questionnaire usefulness scale had good internal consistency at both pre-telehealth training and usage and at post TCP (Cronbach's alpha coefficient pre-usage 0.94 , end of TCP 0.92 ). The ease-of-use scale also had good internal consistency (pre-training and usage 0.92 , end of TCP 0.95).

There were 32 participants and carers who used the telehealth equipment and completed a telehealth acceptance questionnaire prior to telehealth training and usage, and also at the completion of their TCP (up to 12 weeks with telehealth). Age range of participants was 63-95 years (average age 80 years): 20 participants were female and 12 were male; 15 had a carer (47\%) and 17 did not (53\%). Of those carers, $55 \%$ were female and $45 \%$ male; $38 \%$ identified as being a daughter, $25 \%$ as a husband, $20 \%$ as a son, and $17 \%$ as a wife; $68 \%$ lived in the same residence as the participant, and $32 \%$ lived in a separate residence; $90 \%$ reported caring for a participant who had capacity to make their own health care decisions, and 10\% reported caring for someone who did not. Carers were not asked their age. Carers were observationally assessed by BCS staff as having the physical and cognitive capacity to operate the telehealth equipment, complete questionnaires, and to report any issues to staff.

As indicated in Table 2, independent $t$-tests revealed no significant differences in the TAM responses provided by the participants without carers $(n=17)$ or the carers of participants $(n=15)$ for both pre- and post-telehealth usage; there were small to very small effect sizes between those two groups.
The 32 participants and carers recorded a $12 \%$ telehealth reading failure rate. Overall, staff not following up missed or incomplete readings accounted for $33 \%$ of the failed readings, equipment failure for $31 \%$, participant noncompliance accounted for $30 \%$, and user error for $6 \%$.

Due to small sample size, groups were collapsed across the conditions to look at acceptance of in-home telehealth as measured by the telehealth acceptance questionnaire scales of perceived ease of use and usefulness, and to evaluate pretraining and usage acceptance of telehealth as a predictor of future compliance.

Responses to the telehealth acceptance questionnaire usefulness and ease-of-use scales pre equipment training and use, and post-transition care (up to 12 weeks usage), are presented in Table 3.

A paired samples $t$-test was conducted to evaluate whether the perceived ease of use of the telehealth equipment changed significantly after actual usage. There was a statistically significant improvement from pre-training and usage (mean $[\mathrm{M}]=7.48$, standard deviation $[\mathrm{SD}]=2.63$ ) to the end of TCP (up to 12 weeks of usage) $(\mathrm{M}=5.84, \mathrm{SD}=2.17)$, $t(31)=3.76, P=0.001$. There was no significant difference in how useful the participants perceived the equipment to be from pre-training and usage to the end of TCP (up to 12 weeks of usage).

A standard multiple regression was performed to assess the relationship between pre-training and usage acceptance of telehealth as measured by the perceived usefulness and ease of use scales, and future usage compliance. Preliminary analysis identified that assumptions of normality, linearity, multicollinearity, homoscedasticity, and Mahalanobis distance were met. In combination, pre-usage, perceived usefulness, and ease of use accounted for a nonsignificant $17 \%$ of the variance in the usage compliance rate, $R^{2}=0.17$, adjusted $R^{2}=0.11, F(2,29)=2.97, P=0.06$.

Pearson product-moment correlation found a medium positive relationship between the pre-training and usage

Table 2 TAM response differences for participants and carers pre-usage and post-usage (duration TCP)

\begin{tabular}{|c|c|c|c|c|c|c|c|}
\hline & \multicolumn{2}{|c|}{ Participant } & \multicolumn{2}{|c|}{ Carer } & \multirow[t]{2}{*}{$t(30)$} & \multirow[t]{2}{*}{$P$} & \multirow{2}{*}{$\frac{\text { Cohen's }}{\text { d }}$} \\
\hline & $\tilde{\mathbf{n}}$ & SE & $\tilde{\mathbf{n}}$ & SE & & & \\
\hline \multicolumn{8}{|l|}{ Pre-usage } \\
\hline Usefulness & 6.87 & 0.79 & 7.36 & 0.78 & 0.44 & 0.65 & 0.006 \\
\hline Ease of use & 7.42 & 0.76 & 7.55 & 0.55 & 0.13 & 0.89 & 0.001 \\
\hline \multicolumn{8}{|l|}{ Post-usage } \\
\hline Usefulness & 7.68 & 0.69 & 7.54 & 0.69 & -0.11 & 0.91 & 0.001 \\
\hline Ease of use & 6.13 & 0.58 & 5.56 & 0.49 & -0.73 & 0.47 & 0.017 \\
\hline
\end{tabular}

Notes: The guidelines proposed by Cohen for interpreting the $d$ values are $0.0 \mathrm{I}=$ small effect, $0.06=$ moderate effect, and $0.14=$ large effect. Abbreviations: TAM, technology acceptance model; TCP, Transition Care Program. 
Table 3 Acceptance, usefulness, and ease-of-use scales pre-usage and post-transition care

\begin{tabular}{|c|c|c|c|c|c|c|c|c|c|c|c|c|}
\hline \multirow[t]{4}{*}{$\begin{array}{l}\text { Technology acceptance } \\
\text { model statement }\end{array}$} & \multicolumn{2}{|c|}{ Strongly agree } & \multicolumn{2}{|l|}{ Agree } & \multicolumn{2}{|c|}{ Neutral } & \multicolumn{2}{|c|}{ Disagree } & \multicolumn{2}{|c|}{$\begin{array}{l}\text { Strongly } \\
\text { disagree }\end{array}$} & \multicolumn{2}{|c|}{ Mean } \\
\hline & \multicolumn{10}{|l|}{$\mathbf{N}(\%)$} & \multirow[b]{3}{*}{ Pre } & \multirow[b]{3}{*}{ Post } \\
\hline & \multicolumn{2}{|l|}{1} & \multicolumn{2}{|l|}{2} & \multicolumn{2}{|l|}{3} & \multicolumn{2}{|l|}{4} & \multicolumn{2}{|l|}{5} & & \\
\hline & Pre & Post & Pre & Post & Pre & Post & Pre & Post & Pre & Post & & \\
\hline \multicolumn{13}{|l|}{ Usefulness scale } \\
\hline $\begin{array}{l}\text { Using the telehealth equipment will } \\
\text { improve my access to regular testing } \\
\text { of my health condition }\end{array}$ & $15(47)$ & $13(4 \mid)$ & $14(44)$ & $13(4 \mid)$ & $2(6)$ & $4(12)$ & I (3) & $2(6)$ & $0(0)$ & $0(0)$ & 1.66 & 1.84 \\
\hline $\begin{array}{l}\text { Using the telehealth equipment will } \\
\text { make it easier to do regular testing }\end{array}$ & $15(47)$ & $12(37)$ & $12(37)$ & $14(44)$ & $3(9)$ & $4(12)$ & $2(6)$ & $2(6)$ & $0(0)$ & $0(0)$ & 1.75 & 1.87 \\
\hline $\begin{array}{l}\text { Using the telehealth equipment will } \\
\text { save me time in having regular testing }\end{array}$ & $15(47)$ & $12(37)$ & $10(3 \mathrm{I})$ & $10(31)$ & $3(9)$ & $7(22)$ & $4(13)$ & $3(9)$ & $0(0)$ & $0(0)$ & 1.87 & 2.03 \\
\hline $\begin{array}{l}\text { I will find the telehealth equipment } \\
\text { useful in my regular testing }\end{array}$ & $14(44)$ & $12(37)$ & $10(3 \mathrm{I})$ & $13(4 \mid)$ & $7(22)$ & $6(19)$ & I (3) & I (3) & $0(0)$ & $0(0)$ & 1.84 & 1.87 \\
\hline \multicolumn{12}{|l|}{ Ease-of-use scale } & 7.61 \\
\hline $\begin{array}{l}\text { Learning to operate the telehealth } \\
\text { equipment will be easy for me }\end{array}$ & $13(4 \mid)$ & $21(66)$ & II (34) & $9(28)$ & $7(22)$ & $2(6)$ & I (3) & $0(0)$ & $0(0)$ & $0(0)$ & 1.88 & 1.40 \\
\hline $\begin{array}{l}\text { My interaction with the telehealth } \\
\text { equipment will be clear and } \\
\text { understandable }\end{array}$ & $9(28)$ & $17(53)$ & $17(53)$ & $15(47)$ & $5(16)$ & $0(0)$ & I (3) & $0(0)$ & $0(0)$ & $0(0)$ & 1.94 & 1.47 \\
\hline $\begin{array}{l}\text { It will be easy for me to become skilful } \\
\text { at using the telehealth equipment }\end{array}$ & II (34) & $18(56)$ & $17(53)$ & $13(40)$ & $4(13)$ & I (3) & $0(0)$ & $0(0)$ & $0(0)$ & $0(0)$ & 1.78 & 1.47 \\
\hline $\begin{array}{l}\text { I will find the telehealth equipment } \\
\text { easy to use }\end{array}$ & $9(28)$ & I8 (56) & $18(56)$ & $12(38)$ & $5(16)$ & $2(6)$ & $0(0)$ & $0(0)$ & $0(0)$ & $0(0)$ & 1.88 & 1.50 \\
\hline Total & & & & & & & & & & & 7.48 & 5.84 \\
\hline
\end{tabular}

perception of the ease of using the equipment and future usage compliance, $r=0.40, \mathrm{n}=32, P=0.02$. A positive perception of the ease of using the equipment reported prior to training in and using the telehealth equipment was associated with higher future usage compliance.

There was no significant relationship between the perceived equipment usefulness reported at pre-training and usage, and future usage compliance.

\section{Discussion}

This study explored the impact of telehealth on frail older clients of Transition Care and their carers. Various acceptance and usage issues in relation to telehealth, as used by frail older people and their carers, were investigated. This paper reports participant acceptance of in-home telehealth, and evaluates telehealth acceptance as a predictor for usage compliance. The results indicated that before being trained in and using telehealth, the majority of participants had accepted the technology by agreeing it would be useful and easy to use. This acceptance was also reported post-TCP (up to 12 weeks of usage). The perceived ease of use of the telehealth equipment increased significantly from pre-training and usage to post-TCP (up to 12 weeks of usage) $(P=0.001)$. There was no change, (pre-training and usage to post-TCP) in the perceived usefulness of the telehealth equipment. The telehealth acceptance constructs of ease of use and usefulness, at pre-telehealth training and usage, were not a significant predictor of future compliance $(P=0.06)$. Perceived ease of use, at pre-training and usage, had a positive relationship with future compliance $(P=0.02)$.

The reported acceptance of telehealth at both pre- and post-usage, and the result that perceived ease of use increased significantly after equipment usage, is consistent with other findings. Huis et al, in a study utilizing the TAM to explore home telehealth acceptance and compliance in chronic pain management, found that participants with chronic pain had a high acceptance of the telehealth equipment pre- and postusage, and that perceived ease of use increased significantly after usage, whilst perceived usefulness did not. ${ }^{11}$ The present study extends the evidence base by demonstrating that this association is not limited to people with chronic pain, but that the same relationship exists in frail older people with a range of medical conditions that lead to a need for transition care and their carers.

Huis et al concluded that the increase in perceived ease of use may be due to participants finding the equipment easier to use than initially expected. ${ }^{11}$ This could be the case in this present study. However, the increase could also be accounted 
for by the participant's skill at using the telehealth equipment improving with training, resulting in a higher post-training and usage perception of ease of use.

Also, it should be noted that although the perceived usefulness of the equipment did not significantly increase (pre- to post-usage) the majority of participants and carers had already "strongly agreed" or "agreed" to its perceived usefulness at pre-usage. This high level of pre-usage agreement may have influenced the nonsignificant difference in perceived usefulness pre- to post-usage.

Although the acceptance constructs of perceived ease of use and usefulness, at pre-training and usage, were not a significant predictor of future compliance, perceived ease of use at pre-training and usage was positively correlated with future compliance. This significant relationship is important given that the influences and determinants of telehealth compliance are poorly understood. As such, this relationship needs to be further explored. As previously noted, unless the influences and determinants of compliance are known, interventions to improve compliance are unlikely to be successful. ${ }^{9}$ Understanding the contributors to, and influences of, pre-usage perceived ease of use and its relationship with compliance could assist in the development of interventions designed to maintain high usage compliance rates in home telehealth with frail older adults and their carers.

In this study, although telehealth perceived ease of use, at pre-training and usage, could have been influenced by participant low technology skills, it is unlikely that low technology skills also influenced usage compliance. Any participant with low technology skills when completing the telehealth acceptance questionnaire at pre-training and usage had, through subsequent training and assessment by BCS staff, a competent skill level by the time they used the study equipment. This point is supported by the fact that user error to the end of TCP only accounted for $6 \%$ of the failure rate as compared with $30 \%$ for noncompliance. If those using the equipment lacked usage skill during the study, then the user error would have contributed to a higher percentage of the reading failure rate, which it did not. Given this, whilst a low technology usage skill level may have influenced perceived ease of use prior to participants being trained in and using the equipment, it most likely did not impact on usage compliance. This is due to all participants being competent in using the equipment by the time monitoring commenced. Future research would benefit by exploring factors that both influence perceived ease of use at pre-training and usage, and also account for the significant relationship between perceived ease of use at pre-training and usage, and future usage compliance.

This study explored the relationship between telehealth acceptance (measured by the TAM constructs of ease of use and usefulness) and usage compliance. Telehealth acceptance though is not the only factor that has been hypothesized as having a relationship with telehealth usage compliance. Equipment training and support have also been suggested as possibly impacting on differences in compliance rates. ${ }^{17}$ As previously noted, all participants in this study received the same training and had to be competent in using the equipment before they commenced. As such, it is unlikely that training impacted on differences in usage compliance rates. However, differences in the amount of staff support given to each participant during the study could have been influential. If a participant did not ask for help, staff may not have realized that additional support was needed, and this could have influenced participant compliance. Given the importance of understanding usage compliance, future research is needed to explore factors, apart from acceptance, that could influence or be a determinant of compliance, such as support.

The TAM responses analyzed for this paper were provided by either a participant, $n=17(53 \%)$, or a carer of a participant, $n=15(47 \%)$. Although it could be suggested that the differences in age and frailty between carers and participants may have resulted in differences in the reported acceptance of the monitoring system, independent sample $t$-test revealed that for this study this was not likely the case. There were no significant differences in the TAM responses provided by the participants or the carers for both pre- and post-telehealth usage (Table 2), and follow-up analysis revealed that the magnitude of the effect sizes were small to very small.

As previously noted, 32 participants and carers who used the telehealth equipment, and completed TCP, recorded a $12 \%$ telehealth reading failure rate. This failure rate consisted of staff not following up missed or incomplete readings, equipment failure, participant noncompliance, and user error. This paper examined the relationship between pre-usage telehealth acceptance and compliance. Future research would benefit by investigating the relationship, if any, between preusage telehealth acceptance and the other telehealth failure rate variables.

A number of limitations of the study should be noted, in particular the small sample size. The strict exclusion criteria was essential, both to maximize participant safety and to ensure reliable readings. This, in turn, resulted in low eligibility and 
participation rates, which limits the representativeness of this sample and the generalizability of the findings.

The small sample size also reduced the power of the statistical analysis, which may have, in some instances, resulted in an effect being missed; for example, in the regression analysis which approached, but did not reach, statistical significance at $P=0.06$. In addition to this, the small sample size resulted in groups being collapsed. Future research in this area would benefit from a larger sample size, which would increase the power of the statistical analysis and allow for groups to not be collapsed.

It should also be noted that the high rate of usage acceptance could have been influenced by the fact that those who agreed to take part in the study knew that they may possibly receive telehealth equipment. Therefore it is possible that those agreeing to take part were more motivated and had a higher acceptance of the technology. This may have resulted in an over selection of individuals with an established interest and/or comfort in using the equipment.

Eight participants (totaling $42 \%$ of the drop-outs) did not complete the study either because the participant no longer wanted to be part of the study, or their carer, if they had one, no longer wanted them to be part of the study. Unfortunately those participants, or their carer, did not provide a reason for exiting the study beyond the fact that they no longer wished to take part. This is a limitation of the study. However, to determine whether difficulty in using the system was a reason why those participants may have exited, the telehealth reading data were examined. This revealed that all but one of the participants recorded a smaller reading failure rate (which included user error and compliance rates) than the average failure rate of the participants who completed the study. This suggests that the ease of using the equipment was not a likely factor in the reason(s) why they dropped out.

If a reading was missed or incomplete, the BCS data monitoring officer was required to contact the participant (or their carer) to check on the participant's welfare and to determine, if possible, the reason for the missed or incomplete reading. As such, participants were aware that their vital signs were being monitored as they were provided with feedback about this. However, a limitation was that the BCS data monitoring officer did not, in all instances, follow up missed or incomplete readings, and this may have impacted on the participant's perception of the usefulness of the equipment.

Strengths of this study include that it was undertaken in the conditions in which the population of frail older people entering transition care after hospital discharge would be using the equipment. Also, this paper explored potential influences and determinants of home telehealth compliance with older people. As this area of research enquiry is one where there are few data, and it relates to safety, the findings are important.

In conclusion, in what are potentially the largest users of home telehealth, there is currently limited knowledge about the influences and determinants of home telehealth compliance in frail older adults and their carers. As compliance is directly linked to the safe usage of home telehealth, the finding that pre-usage perceived ease of use is related to future compliance is important. Further research is needed to explore not only this relationship but also other potential influences and determinants of home telehealth compliance in this group. Knowledge about the influences and determinants of home telehealth compliance would assist the development of targeted interventions aimed at encouraging high compliance with users who are recording lower reading rates. Given the frailty of this population, and the safety issues relating to usage compliance, this is important.

\section{Acknowledgments}

This study was conducted in conjunction with Baptist Community Services, NSW and ACT, Australia. Funding for the study was provided by the Australian Government Department of Health and Ageing.

\section{Disclosure}

The authors report no conflicts of interest in this work.

\section{References}

1. Australian Government Productivity Commission. Caring for older Australians. Inquiry report. Canberra: Australian Government Productivity Commission; 2011.

2. Bunn $F$. The effects of telephone consultation on triage and healthcare use and patient satisfaction: a systematic review. Br J Gen Pract. 2005;55(521):956-961.

3. Meystre S. The current state of telemonitoring: a comment on the literature. Telemed J E Health. 2005;11(1):63-69.

4. Schofield RS, Kline SE, Schmalfuss CM, et al. Early outcomes of a care coordination-enhanced telehome care program for elderly veterans with chronic heart failure. Telemed J E Health. 2005;11(1):20-27.

5. Bowes A, McColgan G. Smart Technology and Community Care For Older People: Innovation in West Lothian, Scotland. Edinburgh: Age Concern Scotland; 2006.

6. Schlachta-Fairchild L, Elffrink V, Deickman A. Patient safety, telenursing and telehealth. In: Hughes RG, editor. Patient Safety and Quality: an Evidence Based Handbook for Nurses. Rockville, MD: Agency for Healthcare Research and Quality (US); 2008.

7. Wade R, Shaw K, Cartwright C. Factors affecting provision of successful monitoring in home telehealth. Gerontology. Epub January 18, 2012.

8. Roter D, Hall J, Merisca R, Nordstrom B, Cretin D, Svarstad B. Effectiveness of interventions to improve patient compliance: a meta-analysis. Medical Care. 1998;36(8):1138-1161.

9. Kermani F, Davis M. Patient compliance: setting the scene. In: Davis M, Kermani F, editors. Patience Compliance Sweetening the Pill. Abingdon: Ashgate Publishing Group; 2006. 
10. Rahimpour M, Lovell N, Celler B, McCormick J. Patients' perceptions of a home telecare system. Int J Med Inform. 2008;77:486-498.

11. Huis R, Kosterink S, Barbe T, Lindegard A, Marecek T, Vollenbroek M. Relation between patient satisfaction, compliance and the clinical benefit of a teletreatment application for chronic pain. $J$ Telmed Telecare. 2010;16(6):322-328.

12. Huang J. Exploring the acceptance of telecare among senior citizens: an application of back propogation network. Telemed $J$ E Health. 2011;17(2):111-117.

13. Venkatesh V, Smith R. Creation of favourable user perceptions: exploring the role of intrinsic motivation. MIS Quarterly. 1999; 23(2):239-260.
14. Davis F. Perceived usefulness, ease of use, and user acceptance of information technology. MIS Quartlery. 1989;13(3):319-340.

15. Day M, Demiris G, Oliver D, Courtney K, Hensel B. Exploring underutilization of videophones in hospice settings. Telemed $J$ E Health. 2007;13(1):25-31

16. Orruno E, Gagnon M, Asua J, Abdeljelil A. Evaluation of teledermatology adoption by health-care professionals using a modified technology assistance model. J Telmed Telecare. 2011;17(6):303-307.

17. Edwards J, Halawi L. Telemedicine usage in France and the US: an exploratory investigation using the technology acceptance model. Int J Bus Res. 2007;7(5):18-28.

\section{Publish your work in this journal}

Risk Management and Healthcare Policy is an international, peerreviewed, open access journal focusing on all aspects of public health policy, and preventative measures to promote good health and improve morbidity and mortality in the population. The journal welcomes submitted papers covering original research, basic science, clinical \& epidemio-

\section{Dovepress}

logical studies, reviews and evaluations, guidelines, expert opinion and commentary, case reports and extended reports. The manuscript management system is completely online and includes a very quick and fair peerreview system, which is all easy to use. Visit http://www.dovepress.com/ testimonials.php to read real quotes from published authors.

Submit your manuscript here: http://www.dovepress.com/risk-management-and-healthcare-policy-journal 